\title{
Breakfast can Affect Routine Hematology and Coagulation Laboratory Testing: An Evaluation on Behalf of COLABIOCLI WG-PRE-LATAM
}

\author{
Maria Elena Arredondo ${ }^{1, *}$ Eduardo Aranda ${ }^{2, *}$ Rubén Astorga ${ }^{1} \quad$ Lorena Michele Brennan-Bourdon ${ }^{3, *}$ \\ Marise Danielle Campelo ${ }^{4}$ Silvia Flores ${ }^{5, *}$ Claudio Medel $^{1}$ Ignacio Manríquez ${ }^{6}$ Patricia Ochoa ${ }^{7, *}$ \\ Beatriz Varela ${ }^{8, *}$ Carlos Vega Salinas ${ }^{6}$ Gabriel Lima-Oliveira ${ }^{9, *(1)}$
}

${ }^{1}$ BIONET S.A., Santiago, Chile

${ }^{2}$ Laboratory of Thrombosis and Hemostasis, Department of Hematology-Oncology, School of Medicine, Pontificia Universidad Católica de Chile, Santiago, Chile

${ }^{3}$ Clinical Laboratory Network from the State of Jalisco,

Public Health State Laboratory (LESP), Comisión Para la Protección Contra Riesgos Sanitarios del Estado de Jalisco (COPRISJAL),

Guadalajara, Mexico

${ }^{4}$ Clinical Laboratory Bioanalise, Teresina, Piaui, Brazil

5 Universidad Peruana Cayetano Heredia, Lima, Perú

${ }^{6}$ Clínica Dávila y Servicios Médicos S.A., Santiago, Chile

${ }^{7}$ Facultad de Medicina, Universidad Católica de Cuenca, Cuenca, Ecuador

${ }^{8}$ LAC, Montevideo, Uruguay

${ }^{9}$ Section of Clinical Biochemistry, Department of Neurosciences, Biomedicine and Movement Sciences, University of Verona,

Verona, Italy

TH Open 2019;3:e367-e376.
Address for correspondence Gabriel Lima-Oliveira, MSc, PhD, Section of Clinical Biochemistry, Department of Neurosciences, Biomedicine and Movement Sciences, University of Verona, Verona, Italy (e-mail: dr.g.lima.oliveira@gmail.com).

\author{
Abstract \\ Keywords \\ - reproducibility of \\ results \\ - postprandial period \\ - fasting \\ - diagnostic errors \\ - blood specimen \\ collection
}

Laboratories worldwide perform both hematological and coagulation testing on patients avoiding fasting time. In 2017, the Latin America Confederation of Clinical Biochemistry (COLABIOCLI) commissioned the Latin American Working Group for Preanalytical Phase (WG-PRE-LATAM) to study preanalytical variability and establish guidelines for preanalytical procedures to be applied by clinical laboratories and health care professionals. This study, on behalf of COLABIOCLI WG-PRE-LATAM, aims to evaluate the effect of the breakfast on routine hematology and coagulation laboratory testing. We studied 20 healthy volunteers who consumed a breakfast containing a standardized amount of carbohydrates, proteins, and lipids. We collected blood specimens for routine hematology and coagulation laboratory testing before breakfast and 1, 2, and 4 hours thereafter. Significant differences between samples were assessed by the Wilcoxon ranked-pairs test. Statistically significant differences $(p<0.05)$ between basal and 4 hours after the

\footnotetext{
Latin American Working Group for Preanalytical Phase (WGPRE-LATAM) of the Latin America Confederation of Clinical Biochemistry (COLABIOCLI), Montevideo, Uruguay.
}

received

August 30, 2019 accepted after revision October 25, 2019
DOI https://doi.org/

10.1055/s-0039-3401002. ISSN 2512-9465.
๑) 2019 Georg Thieme Verlag KG
Stuttgart · New York

License terms

(c) (1) 
breakfast were observed for red blood cells, hemoglobin, hematocrit, mean corpuscular volume, white blood cells, neutrophils, lymphocytes, monocytes, mean platelet volume, and activated partial thromboplastin time. In conclusion, the significant variations observed in several hematological parameters, and activated partial thromboplastin time due to breakfast feeding demonstrate that the fasting time needs to be carefully considered prior to performing routine hematological and coagulation testing to avoid interpretive mistakes of test results, and to guarantee patient safety. Therefore, COLABIOCLI WG-PRE-LATAM encourages laboratory quality managers to standardize the fasting requirements in their laboratory, i.e., 12 hours.

\section{Introduction}

In 2017, the Latin America Confederation of Clinical Biochemistry (COLABIOCLI) commissioned the Latin American Working Group for Preanalytical Phase (WG-PRE-LATAM) to study preanalytical variability and establish guidelines for preanalytical procedures to be applied by clinical laboratories and health care professionals. The procedures and processes involved in the preanalytical phase are considered the main source of laboratory variability. ${ }^{1,2}$ Providing proper information for patient preparation before laboratory testing is one important issue addressed to accredited laboratories to guarantee patient safety. ${ }^{3}$

Lippi et al, 9 years ago (2010), evaluated the impact of an Italian light meal on hematology testing and evidenced significant variation of several hematological parameters. Therefore, to interpret the results of hematological tests correctly, the fasting time needs to be carefully considered. ${ }^{4}$ However, laboratories worldwide perform both hematological and coagulation testing on patients avoiding fasting time.

This study, on behalf of COLABIOCLI WG-PRE-LATAM, aims to evaluate the effect of the breakfast on routine hematology and coagulation laboratory testing.

\section{Materials and Methods}

A total of 20 healthy volunteers (10 women and 10 men; average age was 42 [23-64] years) were selected from the personnel of the Laboratory BioNet (Santiago, Chile) and included in the study. Informed consent was obtained from all study subjects according to the 2013 Declaration of Helsinki and the protocol was approved by the ethics committee.

A single expert phlebotomist, following the international EFLM-COLABIOCLI recommendations, ${ }^{5}$ performed all venous blood sampling procedures. To eliminate possible blood distribution interferences, all volunteers stayed sitting for 15 minutes. ${ }^{6,7}$ A subcutaneous tissue transilluminator device (Venoscópio IV plus; Duan do Brasil, Brazil) was used to locate a vein on the forearm to prevent venous stasis interference from the use of the tourniquet, ${ }^{8-10}$ and avoid clench. ${ }^{11,12}$

All blood samples were collected respectively into one 3.6mL evacuated tube containing sodium citrate $3.2 \%$, one $3.0-\mathrm{mL}$ evacuated tube containing $\mathrm{K}_{2}$ EDTA, and one 3.0-mL evacuated tube containing $\mathrm{K}_{3}$ EDTA (Vacumed, FL Medical, Torreglia, Italy) using a 20-gauge needle (FL Medical). To eliminate any possible interference due to either the contact phase or tissue factor, approximately $2 \mathrm{~mL}$ of blood were preliminarily collected in a discard tube without additive. The first blood sample was collected between 8:00 a.m. and 8:30 a.m. after a 12-hour overnight fast (i.e., volunteers were not allowed to eat, or drink during this period of time). Moreover, strenuous physical activity was avoided by all volunteers at least 72 hours before blood collection. Volunteers were not smokers and had not intake any medication. Immediately after the first venous blood sampling procedure, the volunteers consumed the breakfast, containing standardized amounts of carbohydrates, protein, and lipids. - Table 1 shows the exact composition of the breakfast. Subsequent venous blood samplings were performed at 1, 2, and 4 hours after breakfast. Each phase of the blood sampling procedure was appropriately standardized, including the use of needles and evacuated tubes from the same type and lot number. No specimens were discarded due to unsatisfactory attempts, for example, problems in locating a suitable vein.

The sample tubes for coagulation assays were left in upright position for 30 minutes at room temperature $\left(20^{\circ} \mathrm{C}\right)$ to ensure complete blood stability before centrifugation. Then, sample tubes were centrifuged at $1,500 \times g$ for 15 minutes at room temperature, according to the instructions provided by the evacuated tube manufacturer (i.e., FL Medical). No samples were either hemolytic or lipemic by visual inspection.

All samples collected were assayed in a single analytical run with the same analyzer according to the manufacturer's specifications and using proprietary reagents. The panel of tests performed is shown in - Tables $\mathbf{2}$ and $\mathbf{3}$. Hematological parameters were assayed on ADIVIA 2120 (Siemens Healthcare Diagnostics Inc., Tarrytown, New York, United States), and on Sysmex XN-1000 (Sysmex Corporation, Kobe, Japan), whereas coagulation tests were performed on ACL TOP 700 (Werfen, Barcelona, Spain) and on Sysmex CA-1500 (Sysmex Corporation). The instruments were calibrated against appropriate proprietary reference standard materials and verified with independent third-party control materials from calibrator materials, as recommended. ${ }^{13}$ The evaluation of the withinrun precision by the internal quality control of the instruments used, showed low coefficients of variation ( $\mathbf{- T a b l e s} \mathbf{2}$ and $\mathbf{3}$ ).

\section{Statistical Analysis}

To assess statistical differences between samples, the Wilcoxon ranked-pairs test was used in agreement with 
Table 1 Nutritional composition of Chilean breakfast

\begin{tabular}{|l|l|l|l|l|l|}
\hline Nutritional composition & Sandwich & Chocolate snack & Yogurt & Orange juice & Total \\
\hline Number (overall weight) & $1(130 \mathrm{~g})$ & $1(45 \mathrm{~g})$ & $1(120 \mathrm{~g})$ & $1(200 \mathrm{~mL})$ & $495 \mathrm{~g}$ \\
\hline Kcal & 384 & 241 & 103 & 50 & 778 \\
\hline Kilojoule (KJ) & 1,607 & 1,008 & 431 & 209 & 3,255 \\
\hline Protein $(\mathrm{g})$ & 17.6 & 2.8 & 3.2 & 0.6 & 24.2 \\
\hline Carbohydrate $(\mathrm{g})$ & 50.3 & 27.0 & 19.0 & 11.2 & 107.5 \\
\hline Total lipids $(\mathrm{g})$ & 12.5 & 14.0 & NA & 0.2 & 28.3 \\
\hline Cholesterol (mg) & 19.2 & NA & & NA & 19.2 \\
\hline
\end{tabular}

Abbreviation: NA, not available.

Table 2 Postprandial variation on complete blood count after Chilean breakfast

\begin{tabular}{|c|c|c|c|c|c|c|c|c|}
\hline \multirow[t]{2}{*}{ Parameters } & \multicolumn{4}{|l|}{$\mathrm{K}_{2}$ EDTA } & \multicolumn{4}{|l|}{$\mathrm{K}_{3}$ EDTA } \\
\hline & Basal & $1 \mathrm{~h}$ & $2 \mathrm{~h}$ & $4 \mathrm{~h}$ & Basal & $1 \mathrm{~h}$ & $2 \mathrm{~h}$ & $4 \mathrm{~h}$ \\
\hline $\begin{array}{l}\text { RBC }\left(10^{12} / \mathrm{L}\right)^{\mathrm{a}} \\
(\mathrm{CVa}=1.05 \%)\end{array}$ & $\begin{array}{l}4.69 \\
{[4.50-4.98]}\end{array}$ & $\begin{array}{l}4.69 \\
{[4.53-5.05]}\end{array}$ & $\begin{array}{l}4.67 \\
{[4.51-4.99]}\end{array}$ & $\begin{array}{l}4.66 \\
{[4.52-4.93]}\end{array}$ & $\begin{array}{l}4.76 \\
{[4.54-5.01]}\end{array}$ & $\begin{array}{l}4.73 \\
{[4.58-5.07]}\end{array}$ & $\begin{array}{l}4.62 \\
{[4.47-5.01]}\end{array}$ & $\begin{array}{l}4.60 \\
{[4.49-4.93]}\end{array}$ \\
\hline$p$-Value & & 0.153 & 0.081 & 0.014 & & 0.940 & $<0.001$ & $<0.001$ \\
\hline$p$-Value $K_{2}$ vs. $K_{3}$ & \multicolumn{8}{|c|}{0.007} \\
\hline $\begin{array}{l}\text { RBC }\left(10^{12} / \mathrm{L}\right)^{\mathrm{b}} \\
(\mathrm{CVa}=0.58 \%)\end{array}$ & $\begin{array}{l}4.76 \\
{[4.55-5.47]}\end{array}$ & $\begin{array}{l}4.75 \\
{[4.61-5.46]}\end{array}$ & $\begin{array}{l}4.70 \\
{[4.56-5.39]}\end{array}$ & $\begin{array}{l}4.66 \\
{[4.50-5.32]}\end{array}$ & $\begin{array}{l}4.76 \\
{[4.53-5.45]}\end{array}$ & $\begin{array}{l}4.76 \\
{[4.59-5.44]}\end{array}$ & $\begin{array}{l}4.72 \\
{[4.53-5.33]}\end{array}$ & $\begin{array}{l}4.68 \\
{[4.50-5.39]}\end{array}$ \\
\hline$p$-Value & & 0.780 & 0.012 & $<0.001$ & & 1.000 & 0.012 & 0.002 \\
\hline$p$-Value $K_{2}$ vs. $K_{3}$ & \multicolumn{8}{|c|}{0.709} \\
\hline $\begin{array}{l}\text { Retic }\left(10^{9} / \mathrm{L}\right)^{\mathrm{a}} \\
(\mathrm{CVa}=4.07 \%)\end{array}$ & $\begin{array}{l}85.8 \\
{[75.1-106]}\end{array}$ & $\begin{array}{l}91.4 \\
{[77.6-108]}\end{array}$ & $\begin{array}{l}87.5 \\
{[71.4-101]}\end{array}$ & $\begin{array}{l}95.0 \\
{[71.0-97.4]}\end{array}$ & $\begin{array}{l}83.9 \\
{[71.8-105]}\end{array}$ & $\begin{array}{l}85.4 \\
{[73.8-104]}\end{array}$ & $\begin{array}{l}86.2 \\
{[69.3-94.7]}\end{array}$ & $\begin{array}{l}87.0 \\
{[66.8-100]}\end{array}$ \\
\hline$p$-Value & & 0.185 & 0.668 & 0.926 & & 0.940 & 0.003 & 0.097 \\
\hline$p$-Value $K_{2}$ vs. $K_{3}$ & \multicolumn{8}{|c|}{0.985} \\
\hline $\begin{array}{l}\text { Retic }\left(10^{9} / \mathrm{L}\right)^{\mathrm{b}} \\
(\mathrm{CVa}=3.21 \%)\end{array}$ & $\begin{array}{l}53.5 \\
{[46.8-73.0]}\end{array}$ & $\begin{array}{l}56.6 \\
{[46.2-69.6]}\end{array}$ & $\begin{array}{l}51.2 \\
{[46.4-68.8]}\end{array}$ & $\begin{array}{l}53.8 \\
{[46.4-72.8]}\end{array}$ & $\begin{array}{l}56.4 \\
{[45.1-76.3]} \\
\end{array}$ & $\begin{array}{l}54.0 \\
{[47.4-72.5]}\end{array}$ & $\begin{array}{l}52.1 \\
{[45.8-73.0]}\end{array}$ & $\begin{array}{l}53.4 \\
{[45.2-69.3]}\end{array}$ \\
\hline$p$-Value & & 0.103 & 0.438 & 0.426 & & 0.330 & 0.209 & 0.116 \\
\hline$p$-Value $K_{2}$ vs. $K_{3}$ & \multicolumn{8}{|c|}{0.670} \\
\hline $\begin{array}{l}\mathrm{Hb}(\mathrm{g} / \mathrm{L})^{\mathrm{a}} \\
(\mathrm{CVa}=0.93 \%)\end{array}$ & $\begin{array}{l}146 \\
{[138-157]}\end{array}$ & $\begin{array}{l}144 \\
{[139-154]}\end{array}$ & $\begin{array}{l}144 \\
{[136-154]}\end{array}$ & $\begin{array}{l}142 \\
{[134-152]}\end{array}$ & $\begin{array}{l}144 \\
{[138-153]}\end{array}$ & $\begin{array}{l}145 \\
{[139-156]}\end{array}$ & $\begin{array}{l}142 \\
{[134-153]}\end{array}$ & $\begin{array}{l}142 \\
{[135-152]}\end{array}$ \\
\hline$p$-Value & & 0.549 & 0.004 & $<0.001$ & & 0.699 & 0.002 & $<0.001$ \\
\hline$p$-Value $K_{2}$ vs. $K_{3}$ & \multicolumn{8}{|c|}{0.043} \\
\hline $\begin{array}{l}\mathrm{Hb}(\mathrm{g} / \mathrm{L})^{\mathrm{b}} \\
(\mathrm{CVa}=0.53 \%)\end{array}$ & $\begin{array}{l}147 \\
{[140-160]}\end{array}$ & $\begin{array}{l}145 \\
{[142-160]}\end{array}$ & $\begin{array}{l}145 \\
{[139-156]}\end{array}$ & $\begin{array}{l}143 \\
{[140-156]}\end{array}$ & $\begin{array}{l}146 \\
{[142-161]}\end{array}$ & $\begin{array}{l}146 \\
{[142-159]}\end{array}$ & $\begin{array}{l}144 \\
{[138-156]}\end{array}$ & $\begin{array}{l}143 \\
{[139-157]}\end{array}$ \\
\hline$p$-Value & & 0.406 & 0.042 & 0.006 & & 0.875 & 0.008 & 0.002 \\
\hline$p$-Value $K_{2}$ vs. $K_{3}$ & \multicolumn{8}{|c|}{0.944} \\
\hline $\begin{array}{l}\operatorname{Hct}(\%)^{\mathrm{a}} \\
(\mathrm{CVa}=1.08 \%)\end{array}$ & $\begin{array}{l}43.6 \\
{[41.8-46.4]}\end{array}$ & $\begin{array}{l}43.7 \\
{[42.2-46.8]}\end{array}$ & $\begin{array}{l}43.6 \\
{[41.3-46.9]}\end{array}$ & $\begin{array}{l}42.9 \\
{[41.0-45.4]}\end{array}$ & $\begin{array}{l}43.4 \\
{[41.8-45.9]}\end{array}$ & $\begin{array}{l}43.6 \\
{[41.6-46.6]}\end{array}$ & $\begin{array}{l}42.2 \\
{[40.4-45.6]}\end{array}$ & $\begin{array}{l}42.1 \\
{[40.6-44.9]}\end{array}$ \\
\hline$p$-Value & & 0.038 & 0.018 & $<0.001$ & & 0.765 & 0.001 & $<0.001$ \\
\hline$p$-Value $K_{2}$ vs. $K_{3}$ & \multicolumn{8}{|c|}{0.013} \\
\hline $\begin{array}{l}\mathrm{Hct}(\%)^{\mathrm{b}} \\
(\mathrm{CVa}=0.54 \%)\end{array}$ & $\begin{array}{l}45.8 \\
{[43.8-49.7]}\end{array}$ & $\begin{array}{l}45.3 \\
{[44.2-49.4]}\end{array}$ & $\begin{array}{l}44.6 \\
{[42.5-48.6]}\end{array}$ & $\begin{array}{l}43.8 \\
{[41.9-47.6]}\end{array}$ & $\begin{array}{l}44.4 \\
{[42.7-48.6]}\end{array}$ & $\begin{array}{l}44.4 \\
{[43.2-48.1]}\end{array}$ & $\begin{array}{l}43.4 \\
{[41.4-47.2]}\end{array}$ & $\begin{array}{l}42.7 \\
{[41.5-47.0]}\end{array}$ \\
\hline$p$-Value & & 0.683 & 0.003 & 0.001 & & 1.000 & 0.002 & 0.001 \\
\hline$p$-Value $K_{2}$ vs. $K_{3}$ & \multicolumn{8}{|c|}{0.001} \\
\hline $\begin{array}{l}\mathrm{MCV}(\mathrm{fL})^{\mathrm{a}} \\
(\mathrm{CVa}=0.32 \%)\end{array}$ & $\begin{array}{l}92.8 \\
{[90.0-95.6]}\end{array}$ & $\begin{array}{l}92.8 \\
{[90.2-95.1]}\end{array}$ & $\begin{array}{l}92.2 \\
{[89.8-94.4]}\end{array}$ & $\begin{array}{l}91.6 \\
{[89.5-94.1]}\end{array}$ & $\begin{array}{l}91.5 \\
{[88.9-94.1]}\end{array}$ & $\begin{array}{l}91.4 \\
{[89.0-93.6]}\end{array}$ & $\begin{array}{l}90.9 \\
{[88.7-92.9]}\end{array}$ & $\begin{array}{l}90.3 \\
{[88.0-92.5]}\end{array}$ \\
\hline$p$-Value & & 0.059 & $<0.001$ & $<0.001$ & & 0.093 & $<0.001$ & $<0.001$ \\
\hline$p$-Value $K_{2}$ vs. $K_{3}$ & \multicolumn{8}{|c|}{$<0.001$} \\
\hline $\begin{array}{l}\mathrm{MCV}(\mathrm{fL})^{\mathrm{b}} \\
(\mathrm{CVa}=0.14 \%)\end{array}$ & $\begin{array}{l}95.0 \\
{[91.1-96.9]}\end{array}$ & $\begin{array}{l}95.2 \\
{[91.1-96.4]}\end{array}$ & $\begin{array}{l}93.8 \\
{[90.3-96.0]}\end{array}$ & $\begin{array}{l}93.0 \\
{[90.0-95.1]}\end{array}$ & $\begin{array}{l}93.2 \\
{[89.4-94.6]}\end{array}$ & $\begin{array}{l}93.2 \\
{[89.3-94.3]}\end{array}$ & $\begin{array}{l}91.8 \\
{[88.4-93.8]}\end{array}$ & $\begin{array}{l}91.4 \\
{[88.0-93.2]}\end{array}$ \\
\hline$p$-Value & & 0.306 & 0.004 & $<0.001$ & & 0.937 & 0.002 & 0.001 \\
\hline$p$-Value $K_{2}$ vs. $K_{3}$ & \multicolumn{8}{|c|}{0.001} \\
\hline
\end{tabular}


Table 2 (Continued)

\begin{tabular}{|c|c|c|c|c|c|c|c|c|}
\hline \multirow[t]{2}{*}{ Parameters } & \multicolumn{4}{|l|}{$\mathrm{K}_{2}$ EDTA } & \multicolumn{4}{|l|}{$\mathrm{K}_{3}$ EDTA } \\
\hline & Basal & $1 \mathrm{~h}$ & $2 \mathrm{~h}$ & $4 \mathrm{~h}$ & Basal & $1 \mathrm{~h}$ & $2 \mathrm{~h}$ & $4 \mathrm{~h}$ \\
\hline $\begin{array}{l}\text { RDW (\%) }{ }^{\mathrm{a}} \\
(\mathrm{CVa}=0.57 \%)\end{array}$ & $\begin{array}{l}13.0 \\
{[12.6-13.3]}\end{array}$ & $\begin{array}{l}13.0 \\
{[12.5-13.3]}\end{array}$ & $\begin{array}{l}12.9 \\
{[12.5-13.3]}\end{array}$ & $\begin{array}{l}13.0 \\
{[12.4-13.2]}\end{array}$ & $\begin{array}{l}13.0 \\
{[12.5-13.3]}\end{array}$ & $\begin{array}{l}13.0 \\
{[12.4-13.3]}\end{array}$ & $\begin{array}{l}12.9 \\
{[12.5-13.2]}\end{array}$ & $\begin{array}{l}12.9 \\
{[12.4-13.2]}\end{array}$ \\
\hline$p$-Value & & 0.479 & 0.064 & 0.592 & & 0.461 & 0.079 & 0.077 \\
\hline$p$-Value $K_{2}$ vs. $K_{3}$ & \multicolumn{8}{|c|}{0.745} \\
\hline $\begin{array}{l}\text { RDW (\%) } \\
(C V a=0.56 \%)\end{array}$ & $\begin{array}{l}13.2 \\
{[12.7-13.7]}\end{array}$ & $\begin{array}{l}13.2 \\
{[12.8-13.6]}\end{array}$ & $\begin{array}{l}13.0 \\
{[12.7-13.5]}\end{array}$ & $\begin{array}{l}13.0 \\
{[12.7-13.5]}\end{array}$ & $\begin{array}{l}13.1 \\
{[12.7-13.6]}\end{array}$ & $\begin{array}{l}13.0 \\
{[12.8-13.5]}\end{array}$ & $\begin{array}{l}13.0 \\
{[12.7-13.4]}\end{array}$ & $\begin{array}{l}13.0 \\
{[12.5-13.4]}\end{array}$ \\
\hline$p$-Value & & 0.507 & 0.012 & 0.011 & & 0.546 & 0.586 & 0.588 \\
\hline$p$-Value $K_{2}$ vs. $K_{3}$ & \multicolumn{8}{|c|}{0.114} \\
\hline $\begin{array}{l}\text { WBC }\left(10^{9} / L\right)^{a} \\
(C V a=1.66 \%)\end{array}$ & $\begin{array}{l}6.09 \\
{[5.15-7.06]}\end{array}$ & $\begin{array}{l}6.20 \\
{[5.25-7.92]} \\
\end{array}$ & $\begin{array}{l}6.80 \\
{[5.87-8.05]}\end{array}$ & $\begin{array}{l}7.12 \\
{[6.11-8.82]}\end{array}$ & $\begin{array}{l}6.36 \\
{[5.14-7.10]} \\
\end{array}$ & $\begin{array}{l}6.44 \\
{[5.55-7.99]}\end{array}$ & $\begin{array}{l}6.72 \\
{[5.86-8.16]}\end{array}$ & $\begin{array}{l}7.18 \\
{[6.15-8.94]} \\
\end{array}$ \\
\hline$p$-Value & & 0.422 & 0.002 & $<0.001$ & & 0.287 & 0.004 & 0.001 \\
\hline$p$-Value $K_{2}$ vs. $K_{3}$ & \multicolumn{8}{|c|}{0.179} \\
\hline $\begin{array}{l}\text { WBC }\left(10^{9} / \mathrm{L}\right)^{\mathrm{b}} \\
(\mathrm{CVa}=1.30 \%)\end{array}$ & $\begin{array}{l}5.99 \\
{[4.93-6.90]}\end{array}$ & $\begin{array}{l}6.27 \\
{[5.54-6.71]}\end{array}$ & $\begin{array}{l}6.53 \\
{[6.07-7.65]}\end{array}$ & $\begin{array}{l}6.97 \\
{[6.50-8.51]}\end{array}$ & $\begin{array}{l}6.03 \\
{[4.92-6.86]}\end{array}$ & $\begin{array}{l}6.32 \\
{[5.56-6.64]}\end{array}$ & $\begin{array}{l}6.73 \\
{[6.12-7.91]}\end{array}$ & $\begin{array}{l}7.05 \\
{[6.46-8.43]}\end{array}$ \\
\hline$p$-Value & & 0.119 & 0.003 & $<0.001$ & & 0.194 & 0.001 & 0.001 \\
\hline$p$-Value $K_{2}$ vs. $K_{3}$ & \multicolumn{8}{|c|}{0.834} \\
\hline $\begin{array}{l}\operatorname{NEU~}\left(10^{9} / \mathrm{L}\right)^{\mathrm{a}} \\
(\mathrm{CVa}=2.27 \%)\end{array}$ & $\begin{array}{l}3.45 \\
{[2.46-4.10]}\end{array}$ & $\begin{array}{l}3.66 \\
{[3.01-4.77]}\end{array}$ & $\begin{array}{l}4.12 \\
{[3.19-4.88]}\end{array}$ & $\begin{array}{l}4.06 \\
{[3.10-5.03]}\end{array}$ & $\begin{array}{l}3.52 \\
{[2.41-4.19]}\end{array}$ & $\begin{array}{l}3.76 \\
{[3.05-5.03]}\end{array}$ & $\begin{array}{l}4.18 \\
{[3.18-4.92]}\end{array}$ & $\begin{array}{l}4.12 \\
{[3.23-5.08]}\end{array}$ \\
\hline$p$-Value & & 0.001 & 0.001 & 0.002 & & 0.001 & 0.001 & 0.002 \\
\hline$p$-Value $K_{2}$ vs. $K_{3}$ & \multicolumn{8}{|c|}{0.023} \\
\hline $\begin{array}{l}\text { NEU }\left(10^{9} / \mathrm{L}\right)^{\mathrm{b}} \\
(\mathrm{CVa}=2.44 \%)\end{array}$ & $\begin{array}{l}3.27 \\
{[2.32-3.83]}\end{array}$ & $\begin{array}{l}3.60 \\
{[2.82-4.08]}\end{array}$ & $\begin{array}{l}4.00 \\
{[3.03-4.66]}\end{array}$ & $\begin{array}{l}4.04 \\
{[2.94-5.16]}\end{array}$ & $\begin{array}{l}3.28 \\
{[2.39-3.86]}\end{array}$ & $\begin{array}{l}3.72 \\
{[2.90-4.17]}\end{array}$ & $\begin{array}{l}3.98 \\
{[2.98-4.82]}\end{array}$ & $\begin{array}{l}4.18 \\
{[2.88-5.15]}\end{array}$ \\
\hline$p$-Value & & 0.003 & 0.001 & 0.002 & & 0.003 & 0.002 & $<0.001$ \\
\hline$p$-Value $K_{2}$ vs. $K_{3}$ & \multicolumn{8}{|c|}{0.571} \\
\hline $\begin{array}{l}\operatorname{LYMP}\left(10^{9} / \mathrm{L}\right)^{\mathrm{a}} \\
(\mathrm{CVa}=3.74 \%)\end{array}$ & $\begin{array}{l}2.04 \\
{[1.76-2.42]} \\
\end{array}$ & $\begin{array}{l}1.87 \\
{[1.45-2.20]}\end{array}$ & $\begin{array}{l}1.91 \\
{[1.68-2.36]}\end{array}$ & $\begin{array}{l}2.30 \\
{[2.03-2.78]}\end{array}$ & $\begin{array}{l}2.01 \\
{[1.80-2.39]}\end{array}$ & $\begin{array}{l}1.86 \\
{[1.43-2.22]}\end{array}$ & $\begin{array}{l}1.90 \\
{[1.66-2.38]}\end{array}$ & $\begin{array}{l}2.30 \\
{[1.97-2.83]}\end{array}$ \\
\hline$p$-Value & & 0.013 & 0.629 & $<0.001$ & & 0.016 & 0.906 & $<0.001$ \\
\hline$p$-Value $K_{2}$ vs. $K_{3}$ & \multicolumn{8}{|c|}{0.663} \\
\hline $\begin{array}{l}\text { LYMP }\left(10^{9} / L\right)^{b} \\
(C V a=1.44 \%)\end{array}$ & $\begin{array}{l}2.07 \\
{[1.76-2.32]}\end{array}$ & $\begin{array}{l}1.98 \\
{[1.49-2.28]}\end{array}$ & $\begin{array}{l}2.06 \\
{[1.83-2.50]}\end{array}$ & $\begin{array}{l}2.40 \\
{[2.18-2.78]}\end{array}$ & $\begin{array}{l}2.04 \\
{[1.80-2.39]}\end{array}$ & $\begin{array}{l}1.94 \\
{[1.44-2.24]}\end{array}$ & $\begin{array}{l}2.13 \\
{[1.86-2.52]}\end{array}$ & $\begin{array}{l}2.22 \\
{[2.15-2.88]}\end{array}$ \\
\hline$p$-Value & & 0.132 & 0.543 & $<0.001$ & & 0.119 & 0.315 & $<0.001$ \\
\hline$p$-Value $K_{2}$ vs. $K_{3}$ & \multicolumn{8}{|c|}{0.551} \\
\hline $\begin{array}{l}\text { Mono }\left(10^{9} / \mathrm{L}\right)^{\mathrm{a}} \\
(\mathrm{CVa}=6.27 \%)\end{array}$ & $\begin{array}{l}0.34 \\
{[0.29-0.43]}\end{array}$ & $\begin{array}{l}0.32 \\
{[0.26-0.36]}\end{array}$ & $\begin{array}{l}0.36 \\
{[0.32-0.42]}\end{array}$ & $\begin{array}{l}0.36 \\
{[0.33-0.42]}\end{array}$ & $\begin{array}{l}0.36 \\
{[0.32-0.44]}\end{array}$ & $\begin{array}{l}0.32 \\
{[0.29-0.42]}\end{array}$ & $\begin{array}{l}0.36 \\
{[0.32-0.42]}\end{array}$ & $\begin{array}{l}0.38 \\
{[0.33-0.42]}\end{array}$ \\
\hline$p$-Value & & 0.014 & 0.022 & 0.024 & & 0.139 & 0.920 & 0.170 \\
\hline$p$-Value $K_{2}$ vs. $K_{3}$ & \multicolumn{8}{|c|}{0.217} \\
\hline $\begin{array}{l}\text { Mono }\left(10^{9} / \mathrm{L}\right)^{\mathrm{b}} \\
(\mathrm{CVa}=4.34 \%)\end{array}$ & $\begin{array}{l}0.44 \\
{[0.40-0.53]}\end{array}$ & $\begin{array}{l}0.40 \\
{[0.34-0.47]}\end{array}$ & $\begin{array}{l}0.46 \\
{[0.41-0.56]}\end{array}$ & $\begin{array}{l}0.52 \\
{[0.42-0.57]}\end{array}$ & $\begin{array}{l}0.40 \\
{[0.36-0.53]}\end{array}$ & $\begin{array}{l}0.36 \\
{[0.32-0.47]}\end{array}$ & $\begin{array}{l}0.49 \\
{[0.39-0.57]}\end{array}$ & $\begin{array}{l}0.50 \\
{[0.39-0.59]}\end{array}$ \\
\hline$p$-Value & & 0.052 & 0.221 & 0.004 & & 0.220 & 0.033 & 0.007 \\
\hline$p$-Value $K_{2}$ vs. $K_{3}$ & \multicolumn{8}{|c|}{0.021} \\
\hline
\end{tabular}

Simundic's ${ }^{14}$ recommendations regarding sample size (i.e., less than 30 samples), with a licensed statistical software (GraphPad Prism version 5.01, La Jolla, California, United States). The level of statistical significance was set at $p<0.05$. The mean percentage difference in each test parameter with statistical significance was calculated using the formula:

Mean percentage difference $=[(\times \mathrm{h}$ after breakfast - basal $) / \times$ h after breakfast] $\times 100$

Finally, the mean percentage differences from blood samples at 1,2, and 4 hours after breakfast were compared with the desirable specifications for imprecision (DSI) derived from biological variation. ${ }^{15}$ DSI was used as our criterion of acceptance in lipemia analytical interference testing, then interferograms were provided for each laboratory parameter with a significant difference between basal and $\times \mathrm{h}$ after the breakfast.

\section{Results}

The results of this investigation are presented as median (interquartile range) in - Tables $\mathbf{2}$ and $\mathbf{3}$. Statistically significant differences $(p<0.05)$ between basal and 4 hours after the breakfast were observed for red blood cell (RBC), hemoglobin $(\mathrm{Hb})$, hematocrit $(\mathrm{Hct})$, mean corpuscular 
Table 2 (Continued)

\begin{tabular}{|c|c|c|c|c|c|c|c|c|}
\hline \multirow[t]{2}{*}{ Parameters } & \multicolumn{4}{|l|}{$\mathrm{K}_{2}$ EDTA } & \multicolumn{4}{|l|}{$\mathrm{K}_{3}$ EDTA } \\
\hline & Basal & $1 \mathrm{~h}$ & $2 \mathrm{~h}$ & $4 \mathrm{~h}$ & Basal & $1 \mathrm{~h}$ & $2 \mathrm{~h}$ & $4 \mathrm{~h}$ \\
\hline $\begin{array}{l}\operatorname{Eos}\left(10^{9} / \mathrm{L}\right)^{\mathrm{a}} \\
(\mathrm{CVa}=9.10 \%)\end{array}$ & $\begin{array}{l}0.20 \\
{[0.11-0.27]}\end{array}$ & $\begin{array}{l}0.18 \\
{[0.10-0.24]}\end{array}$ & $\begin{array}{l}0.20 \\
{[0.10-0.28]}\end{array}$ & $\begin{array}{l}0.22 \\
{[0.10-0.25]}\end{array}$ & $\begin{array}{l}0.22 \\
{[0.11-0.28]}\end{array}$ & $\begin{array}{l}0.20 \\
{[0.10-0.28]}\end{array}$ & $\begin{array}{l}0.18 \\
{[0.10-0.26]}\end{array}$ & $\begin{array}{l}0.20 \\
{[0.10-0.26}\end{array}$ \\
\hline$p$-Value & & 0.161 & 0.499 & 1.000 & & 0.147 & 0.053 & 0.528 \\
\hline$p$-Value $K_{2}$ vs. $K_{3}$ & \multicolumn{8}{|c|}{0.300} \\
\hline $\begin{array}{l}\text { Eos }\left(10^{9} / \mathrm{L}\right)^{\mathrm{b}} \\
(\mathrm{CVa}=8.18 \%)\end{array}$ & $\begin{array}{l}0.22 \\
{[0.11-0.32]}\end{array}$ & $\begin{array}{l}0.20 \\
{[0.07-0.26]}\end{array}$ & $\begin{array}{l}0.23 \\
{[0.07-0.28]}\end{array}$ & $\begin{array}{l}0.21 \\
{[0.08-0.26]}\end{array}$ & $\begin{array}{l}0.20 \\
{[0.10-0.30]}\end{array}$ & $\begin{array}{l}0.20 \\
{[0.06-2.25]}\end{array}$ & $\begin{array}{l}0.23 \\
{[0.06-0.29]}\end{array}$ & $\begin{array}{l}0.22 \\
{[0.07-0.30}\end{array}$ \\
\hline$p$-Value & & 0.248 & 0.550 & 0.683 & & 0.184 & 0.476 & 0.753 \\
\hline$p$-Value $K_{2}$ vs. $K_{3}$ & \multicolumn{8}{|c|}{0.784} \\
\hline $\begin{array}{l}\text { Baso }\left(10^{9} / \mathrm{L}\right)^{\mathrm{a}} \\
(\mathrm{CVa}=15.2 \%)\end{array}$ & $\begin{array}{l}0.04 \\
{[0.03-0.05]}\end{array}$ & $\begin{array}{l}0.04 \\
{[0.03-0.05]}\end{array}$ & $\begin{array}{l}0.04 \\
{[0.03-0.05]}\end{array}$ & $\begin{array}{l}0.04 \\
{[0.03-0.05]}\end{array}$ & $\begin{array}{l}0.04 \\
{[0.03-0.05]}\end{array}$ & $\begin{array}{l}0.04 \\
{[0.04-0.05]}\end{array}$ & $\begin{array}{l}0.04 \\
{[0.03-0.05]}\end{array}$ & $\begin{array}{l}0.04 \\
{[0.03-0.05}\end{array}$ \\
\hline$p$-Value & & 0.264 & 0.229 & 0.219 & & 0.796 & 0.954 & 0.837 \\
\hline$p$-Value $K_{2}$ vs. $K_{3}$ & \multicolumn{8}{|c|}{0.209} \\
\hline $\begin{array}{l}\text { Baso }\left(10^{9} / \mathrm{L}\right)^{\mathrm{b}} \\
(\mathrm{CVa}=18.3 \%)\end{array}$ & $\begin{array}{l}0.04 \\
{[0.03-0.05]}\end{array}$ & $\begin{array}{l}0.04 \\
{[0.03-0.04]}\end{array}$ & $\begin{array}{l}0.04 \\
{[0.03-0.05]}\end{array}$ & $\begin{array}{l}0.04 \\
{[0.03-0.06]}\end{array}$ & $\begin{array}{l}0.04 \\
{[0.03-0.05]} \\
\end{array}$ & $\begin{array}{l}0.04 \\
{[0.03-0.06]}\end{array}$ & $\begin{array}{l}0.04 \\
{[0.04-0.05]}\end{array}$ & $\begin{array}{l}0.04 \\
{[0.03-0.05}\end{array}$ \\
\hline$p$-Value & & 0.722 & 0.611 & 0.548 & & 0.781 & 0.257 & 0.187 \\
\hline$p$-Value $K_{2}$ vs. $K_{3}$ & \multicolumn{8}{|c|}{1.000} \\
\hline $\begin{array}{l}\text { Luc }\left(10^{9} / \mathrm{L}\right)^{\mathrm{a}} \\
(\mathrm{CVa}=11.5 \%)\end{array}$ & $\begin{array}{l}0.11 \\
{[0.09-0.12]}\end{array}$ & $\begin{array}{l}0.09 \\
{[0.07-0.10]}\end{array}$ & $\begin{array}{l}0.10 \\
{[0.08-0.12]}\end{array}$ & $\begin{array}{l}0.13 \\
{[0.11-0.15]}\end{array}$ & $\begin{array}{l}0.09 \\
{[0.09-0.10]}\end{array}$ & $\begin{array}{l}0.08 \\
{[0.06-0.09]}\end{array}$ & $\begin{array}{l}0.09 \\
{[0.08-0.11]}\end{array}$ & $\begin{array}{l}0.12 \\
{[0.10-0.13}\end{array}$ \\
\hline$p$-Value & & 0.002 & 0.244 & 0.002 & & 0.017 & 0.417 & 0.004 \\
\hline$p$-Value $K_{2}$ vs. $K_{3}$ & \multicolumn{8}{|c|}{0.003} \\
\hline $\begin{array}{l}\text { IG }\left(10^{9} / \mathrm{L}\right)^{\mathrm{b}} \\
(\mathrm{CVa}=35.1 \%)\end{array}$ & $\begin{array}{l}0.02 \\
{[0.01-0.03]}\end{array}$ & $\begin{array}{l}0.02 \\
{[0.02-0.03]}\end{array}$ & $\begin{array}{l}0.03 \\
{[0.02-0.04]}\end{array}$ & $\begin{array}{l}0.02 \\
{[0.02-0.04]}\end{array}$ & $\begin{array}{l}0.02 \\
{[0.01-0.03]}\end{array}$ & $\begin{array}{l}0.02 \\
{[0.02-0.03]}\end{array}$ & $\begin{array}{l}0.02 \\
{[0.02-0.03]}\end{array}$ & $\begin{array}{l}0.02 \\
{[0.02-0.03}\end{array}$ \\
\hline$p$-Value & & 0.608 & 0.053 & 0.061 & & 0.111 & 0.674 & 0.193 \\
\hline$p$-Value $K_{2}$ vs. $K_{3}$ & \multicolumn{8}{|c|}{0.803} \\
\hline $\begin{array}{l}\text { PLT }\left(10^{9} / \mathrm{L}\right)^{\mathrm{a}} \\
(\mathrm{CVa}=2.43 \%)\end{array}$ & $\begin{array}{l}274 \\
{[240-323]}\end{array}$ & $\begin{array}{l}278 \\
{[247-332]}\end{array}$ & $\begin{array}{l}273 \\
{[246-323]}\end{array}$ & $\begin{array}{l}266 \\
{[253-328]}\end{array}$ & $\begin{array}{l}261 \\
{[245-331]}\end{array}$ & $\begin{array}{l}273 \\
{[247-324]}\end{array}$ & $\begin{array}{l}275 \\
{[245-327]}\end{array}$ & $\begin{array}{l}270 \\
{[253-322]}\end{array}$ \\
\hline$p$-Value & & 0.002 & 0.057 & 0.016 & & 0.100 & 0.332 & 0.093 \\
\hline$p$-Value $K_{2}$ vs. $K_{3}$ & \multicolumn{8}{|c|}{0.370} \\
\hline $\begin{array}{l}\text { PLT }\left(10^{9} / \mathrm{L}\right)^{\mathrm{b}} \\
(\mathrm{CVa}=0.66 \%)\end{array}$ & $\begin{array}{l}247 \\
{[205-301]}\end{array}$ & $\begin{array}{l}264 \\
{[217-305]}\end{array}$ & $\begin{array}{l}254 \\
{[213-303]}\end{array}$ & $\begin{array}{l}252 \\
{[222-304]}\end{array}$ & $\begin{array}{l}256 \\
{[210-300]}\end{array}$ & $\begin{array}{l}258 \\
{[222-311]}\end{array}$ & $\begin{array}{l}258 \\
{[216-307]}\end{array}$ & $\begin{array}{l}258 \\
{[224-311]}\end{array}$ \\
\hline$p$-Value & & 0.006 & 0.027 & 0.109 & & 0.258 & 0.826 & 0.456 \\
\hline$p$-Value $K_{2}$ vs. $K_{3}$ & \multicolumn{8}{|c|}{0.051} \\
\hline $\begin{array}{l}\mathrm{MPV}(\mathrm{fL})^{\mathrm{a}} \\
(\mathrm{CVa}=1.88 \%)\end{array}$ & $\begin{array}{l}8.20 \\
{[7.52-9.00]}\end{array}$ & $\begin{array}{l}7.95 \\
{[7.42-8.68]}\end{array}$ & $\begin{array}{l}7.65 \\
{[7.20-8.50]}\end{array}$ & $\begin{array}{l}7.65 \\
{[7.02-8.65]}\end{array}$ & \begin{tabular}{|l|}
8.25 \\
{$[7.40-8.78]$} \\
\end{tabular} & $\begin{array}{l}7.85 \\
{[7.35-8.75]}\end{array}$ & $\begin{array}{l}7.60 \\
{[7.22-8.58]}\end{array}$ & $\begin{array}{l}7.55 \\
{[7.02-8.48}\end{array}$ \\
\hline$p$-Value & & $<0.001$ & $<0.001$ & $<0.001$ & & 0.024 & $<0.001$ & $<0.001$ \\
\hline$p$-Value $K_{2}$ vs. $K_{3}$ & \multicolumn{8}{|c|}{0.458} \\
\hline $\begin{array}{l}\mathrm{MPV}(\mathrm{fL})^{\mathrm{b}} \\
(\mathrm{CVa}=0.87 \%)\end{array}$ & $\begin{array}{l}9.85 \\
{[9.08-10.8]}\end{array}$ & $\begin{array}{l}9.75 \\
{[8.98-10.8]}\end{array}$ & $\begin{array}{l}9.80 \\
{[9.08-10.8]}\end{array}$ & $\begin{array}{l}9.90 \\
{[9.08-10.9]}\end{array}$ & $\begin{array}{l}10.2 \\
{[9.28-11.0]}\end{array}$ & $\begin{array}{l}10.1 \\
{[9.18-11.0]}\end{array}$ & $\begin{array}{l}10.1 \\
{[9.40-11.0]}\end{array}$ & $\begin{array}{l}10.2 \\
{[9.28-11.0}\end{array}$ \\
\hline$p$-Value & & 0.784 & 1.000 & 0.064 & & 0.436 & 1.000 & 1.000 \\
\hline$p$-Value $K_{2}$ vs. $K_{3}$ & \multicolumn{8}{|c|}{0.001} \\
\hline
\end{tabular}

Abbreviations: Baso, basophils; CVa, analytical coefficient of variation; Eos, eosinophils; Hb, hemoglobin; Hct, hematocrit; IG, immature granulocyte; Luc, large unstained cells; LYMP, lymphocytes; MCV, mean corpuscular volume; Mono, monocytes; MPV, mean platelet volume; NEU, neutrophils; PLT, platelet count; RBC, red blood cell count; RDW, RBC distribution width; Retic, reticulocyte; WBC, white blood cell count.

${ }^{a}$ Hematological parameter was assayed on ADIVIA 2120 (Siemens Healthcare Diagnostics Inc, Tarrytown, New York, United States).

${ }^{b}$ Hematological parameter was assayed on Sysmex XN-1000 (Sysmex Corporation, Kobe, Japan).

Note: Results are presented as median [interquartile range]. $p$-Value represents the significance by Wilcoxon ranked-pairs test. Bold $p$-values indicate statistical significance $(p<0.05)$.

volume (MCV), white blood cell (WBC), neutrophil (NEU), lymphocyte (LYMP), monocyte (MONO), mean platelet volume (MPV), and activated partial thromboplastin time (aPTT) (- Fig. 1). In regard to $K_{2}$ EDTA versus $K_{3}$ EDTA, statistically significant differences $(p<0.05)$ were observed for RBC, Hb, NEU, and Luc in samples assayed on Advia 2120 (Siemens Healthcare, GmbH); MONO and MPV in samples tested on Sysmex XN-1000 (Sysmex Corporation), whereas Hct and MCV values were different in both instruments (-Table 2; -Fig. 1). 
e372 Breakfast can Affect Routine Hematology and Coagulation Laboratory Testing Arredondo et al.

Table 3 Postprandial variation on routine coagulation testing after Chilean breakfast

\begin{tabular}{|c|c|c|c|c|}
\hline Tests & Basal & $1 \mathrm{~h}$ & $2 \mathrm{~h}$ & $4 \mathrm{~h}$ \\
\hline $\begin{array}{l}\mathrm{aPTT}(\mathrm{s})^{\mathrm{a}} \\
(\mathrm{CVa}=1.56 \%)\end{array}$ & $\begin{array}{l}28.9 \\
{[27.8-29.9]}\end{array}$ & $\begin{array}{l}29.2 \\
{[27.8-30.5]}\end{array}$ & $\begin{array}{l}27.6 \\
{[27.8-30.2]}\end{array}$ & $\begin{array}{l}29.2 \\
{[28.0-30.2]}\end{array}$ \\
\hline$p$-Value & & 0.111 & 0.034 & 0.037 \\
\hline $\begin{array}{l}\text { aPTT }(s)^{\mathrm{b}} \\
(\mathrm{CVa}=5.04 \%)\end{array}$ & $\begin{array}{l}34.4 \\
{[33.0-36.4]}\end{array}$ & $\begin{array}{l}34.6 \\
{[32.7-36.2]}\end{array}$ & $\begin{array}{l}33.4 \\
{[32.6-35.1]}\end{array}$ & $\begin{array}{l}33.9 \\
{[32.3-34.9]}\end{array}$ \\
\hline$p$-Value & & 0.334 & 0.033 & 0.007 \\
\hline $\begin{array}{l}\mathrm{PT}(\mathrm{s})^{\mathrm{a}} \\
(\mathrm{CVa}=1.73 \%)\end{array}$ & $\begin{array}{l}11.2 \\
{[10.6-11.5]}\end{array}$ & $\begin{array}{l}11.2 \\
{[10.7-11.4]}\end{array}$ & $\begin{array}{l}11.0 \\
{[10.6-11.4]}\end{array}$ & $\begin{array}{l}11.0 \\
{[10.6-11.3]}\end{array}$ \\
\hline$p$-Value & & 0.693 & 0.704 & 0.307 \\
\hline $\begin{array}{l}\mathrm{PT}(\mathrm{s})^{\mathrm{b}} \\
(\mathrm{CVa}=2.00 \%)\end{array}$ & $\begin{array}{l}11.2 \\
{[11.1-11.5]}\end{array}$ & $\begin{array}{l}11.3 \\
{[11.0-11.4]}\end{array}$ & $\begin{array}{l}11.0 \\
{[11.0-11.4]}\end{array}$ & $\begin{array}{l}11.0 \\
{[10.9-11.2]}\end{array}$ \\
\hline$p$-Value & & 0.220 & 0.069 & 0.097 \\
\hline $\begin{array}{l}\text { Fib (mg/dL) } \\
(\mathrm{CVa}=4.50 \%)\end{array}$ & $\begin{array}{l}304 \\
{[285-354]}\end{array}$ & $\begin{array}{l}304 \\
{[294-351]}\end{array}$ & $\begin{array}{l}302 \\
{[281-340]}\end{array}$ & $\begin{array}{l}306 \\
{[289-350]}\end{array}$ \\
\hline$p$-Value & & 0.571 & 0.010 & 0.687 \\
\hline $\begin{array}{l}\text { Fib (mg/dL) } \\
(\mathrm{CVa}=7.19 \%)\end{array}$ & $\begin{array}{l}254 \\
{[226-288]}\end{array}$ & $\begin{array}{l}254 \\
{[231-286]}\end{array}$ & $\begin{array}{l}246 \\
{[223-279]}\end{array}$ & $\begin{array}{l}254 \\
{[225-289]}\end{array}$ \\
\hline$p$-Value & & 0.552 & $<0.001$ & 0.332 \\
\hline $\begin{array}{l}\text { AT }(\%)^{\mathrm{a}} \\
(\mathrm{CVa}=2.60 \%)\end{array}$ & $\begin{array}{l}112 \\
{[106-116]}\end{array}$ & $\begin{array}{l}112 \\
{[108-118]}\end{array}$ & $\begin{array}{l}112 \\
{[106-118]}\end{array}$ & $\begin{array}{l}114 \\
{[106-121]}\end{array}$ \\
\hline$p$-Value & & 0.389 & 0.050 & 0.004 \\
\hline $\begin{array}{l}\mathrm{PC}(\%)^{\mathrm{a}} \\
(\mathrm{CVa}=1.40 \%)\end{array}$ & $\begin{array}{l}102 \\
{[93.0-124]}\end{array}$ & $\begin{array}{l}103 \\
{[96-128]}\end{array}$ & $\begin{array}{l}103 \\
{[94.5-124]}\end{array}$ & $\begin{array}{l}102 \\
{[95.8-132]}\end{array}$ \\
\hline$p$-Value & & 0.143 & 0.217 & 0.819 \\
\hline $\begin{array}{l}\text { Free PS (\%) }^{\mathrm{a}} \\
(\mathrm{CVa}=6.51)\end{array}$ & $\begin{array}{l}103 \\
{[93.7-108]}\end{array}$ & $\begin{array}{l}103 \\
{[95.4-108]}\end{array}$ & $\begin{array}{l}106 \\
{[95.2-107]}\end{array}$ & $\begin{array}{l}106 \\
{[99.6-110]}\end{array}$ \\
\hline$p$-Value & & 0.542 & $<0.001$ & $<0.001$ \\
\hline
\end{tabular}

Abbreviations: aPTT, activated partial thromboplastin time; AT, antithrombin; CVa, analytical coefficient of variation; Fib, fibrinogen; PC, protein C; PS, protein S; PT, prothrombin time.

${ }^{a}$ Coagulation test was performed on ACL TOP 700 (Werfen, Barcelona, Spain).

${ }^{b}$ Coagulation test was performed on Sysmex CA-1500 (Sysmex Corporation).

Note: Results are presented as median [interquartile range]. $p$-Value represents the significance by Wilcoxon ranked-pairs test. Bold $p$-values indicate statistical significance $(p<0.05)$.

\section{Discussion}

Significant decreases were observed for RBC, Hb, Hct, and MCV 2 hours after breakfast and no return to baseline in the following 4 hours after food intake (-Table 2, - Fig. 1A-D). A study performed in starved rats reported increases in $\mathrm{Hb}$, Hct, and RBC counts because of the hemoconcentration/ dehydration effects caused by the greatly reduced water intake during fasting time. ${ }^{16}$ Consequently, we can explain our RBC, Hb, and Hct results by the hemodiluition/rehydration of the volunteers during breakfast, since they consumed $200 \mathrm{~mL}$ of orange juice and $120 \mathrm{~g}$ of yogurt (-Table 1). An unanswered question is whether subjects who consume fixed volume of plain water 2 or 4 hours before blood collection impact on this kind of variability. A report by Unger et al, evaluated the effect of water ingestion $(300 \mathrm{~mL}) 1$ hour before blood collection, concluding that $300 \mathrm{~mL}$ of water does not impact on routine hematological parameters. ${ }^{17}$
However, after breakfast, during the production of hydrochloric acid by the parietal cells in the stomach, these cells extract chloride anions, carbon dioxide, water, and sodium cations from the blood plasma, and in turn, release bicarbonate back into the plasma after its formation from carbon dioxide and water constituents. The bicarbonate content causes the venous blood leaving the stomach to be more alkaline than the arterial blood delivered to it; producing a temporary increase in $\mathrm{pH}$, the phenomenon known as "alkaline tide." ${ }^{18-20}$ Thus, the alkaline tide can directly affect MCV, then the decrease observed (-Table 2; - Fig. 1D) can be attributed to the efflux of electrolytes and water from erythrocytes.

Postprandial leukocytosis was properly evidenced more than 85 years ago (1932), ${ }^{21}$ revisited by Van Oostrom et al, ${ }^{22}$ and experimentally demonstrated by Lippi et al. ${ }^{4}$ Moreover, the ingestion of a meal increases interleukin-6, the major chemokine responsible for LYMP recruitment. ${ }^{23}$ MONOs are 

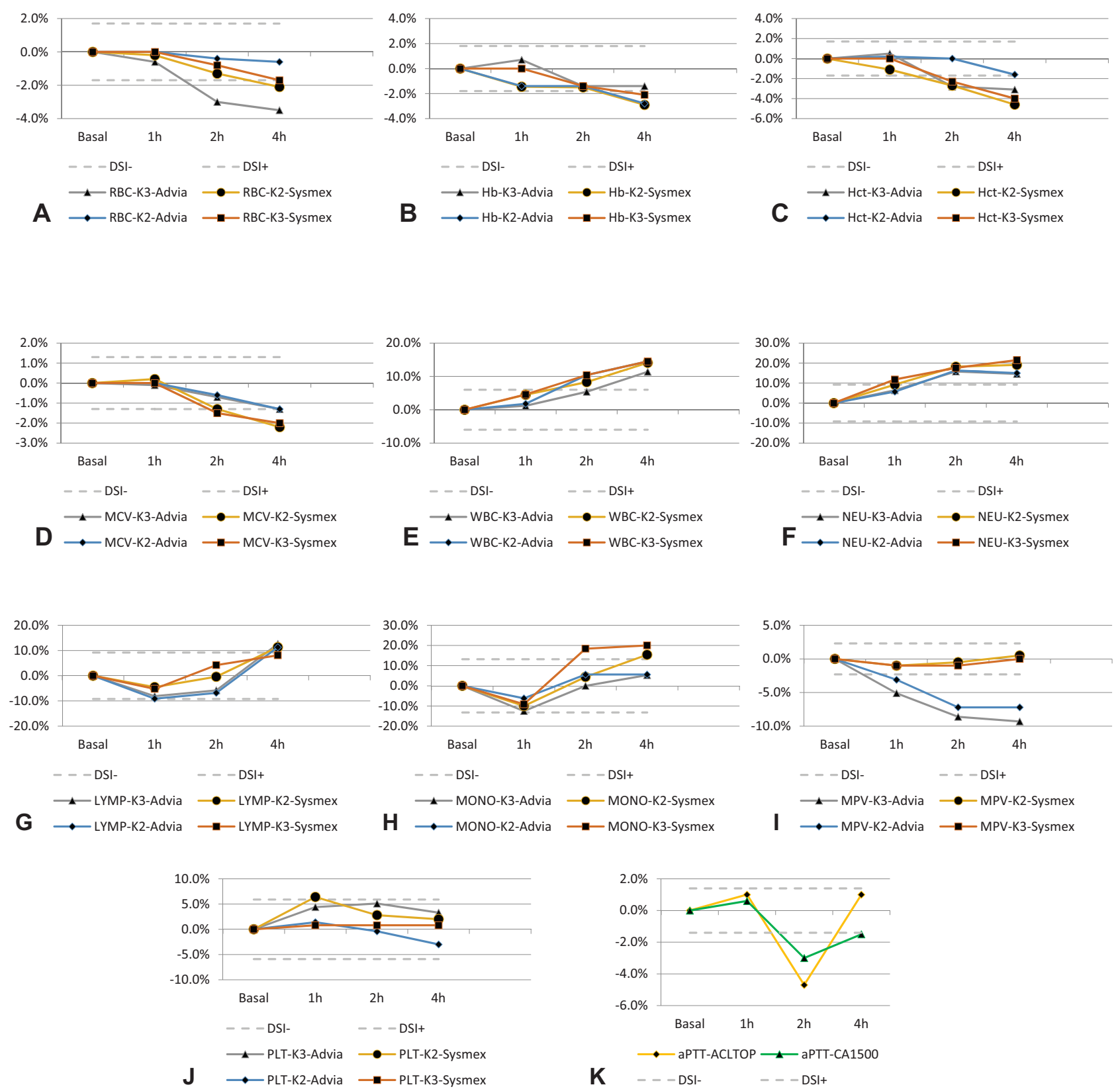

Fig. 1 Interferograms. (A) red blood cell (RBC); (B) hemoglobin (Hb); (C) hematocrit (Hct); (D) mean corpuscular volume (MCV); (E) white blood cell (WBC); (F) neutrophil (NEU); (G) lymphocyte (LYMP); (H) monocyte (MONO); (I) mean platelet volume (MPV); (J) platelet (PLT); and (K) activated partial thromboplastin time (aPTT). Hours after the breakfast ( $x$-axis) are plotted against bias values ( $y$-axis). Solid line, bias. Dashed lines, acceptable criteria based on desirable specification for imprecision (DSI) derived from biologic variation.

activated after food intake. ${ }^{24,25}$ Our results are in agreement with these previous studies; we observed that WBC count and NEU progressively increased 2 and 4 hours after breakfast ( - Table 2; - Fig. 1E, F), whereas LYMP and MONO decreased 1 hour after breakfast and progressively increased 2 and 4 hours after breakfast (-Table 2, - Fig. 1G, H). According to Klop et al, postprandial leukocyte activation is accompanied by temporary changes in leukocyte cell population data, similarly to changes observed during various infections. ${ }^{26}$ However, physicians still request complete blood count $(\mathrm{CBC})$ without advising patients regarding the required fasting time. Furthermore, laboratory medicine professionals, motivated to eliminate fasting time based on their convenience, ${ }^{27}$ must be aware that this represents more risks than benefits for patients. ${ }^{28}$

Postprandial lipemia is associated with changes in inflammatory and thrombotic processes that are known to be important in the development of atherosclerosis. ${ }^{29,30}$ Platelet (PLT) function and activation after breakfast was demonstrated by MPV, without significant changes in PLT count 
(-Table 2; - Fig. 1I, J). In our opinion, MPV results from samples collected avoiding fasting time could improperly lead physicians to consider that as a possible risk factor of microvascular or macrovascular diseases. The routine laboratory coagulation testing including aPTT, for monitoring unfractionated heparin, ${ }^{31}$ could be jeopardized by breakfast (-Table 3; - Fig. 1K). Thus, fasting time should be required before hemostasis testing.

In our study design, each volunteer was devised to be her/ his own control (i.e., the results from 1, 2, and 4 hours after breakfast were compared with basal results of the same individual). Indeed, this kind of study design-a case-crossover study-is most suitable for outcomes where the induction time is short, like our evaluation of breakfast impact on laboratory test results. ${ }^{32}$ In fact, in a case-crossover study, only cases showing discordant exposure status in the case/ control window-of-time contribute to the effect and thus to the measure estimation. Because cases and controls are the same individuals, the problem of between-person confounders-that anyway exists with a control group-being constant the characteristics, do not occur. ${ }^{33}$ Therefore, our design minimizes variability that could jeopardize the preanalytical evaluation on hematology and coagulation laboratory testing. On the other hand, large data collection is not recommended to evaluate preanalytical variability-for example, assessing fasted and nonfasted subjects using questionnaires during weeks, months, or years then correlating these information with their laboratory results-since this kind of study design appears unable to control and exclude other sources of variability as:

- Phlebotomists: each professional that performs blood collection by venipuncture has her/his own mean time of tourniquet application, and this application time is not standardized by institution, also when following international guidelines. ${ }^{34-36}$

- Patient posture: the change from supine to sitting position caused clinically significant increases in the $\mathrm{Hb}$, Hct, and RBC count. Furthermore, the change from supine to standing caused clinically significant increases in the $\mathrm{Hb}$, Hct, RBC, WBC, NEU, LYMP, basophil, PLT, and MPV counts, and the change from sitting to standing caused clinically significant increases in $\mathrm{Hb}, \mathrm{Hct}, \mathrm{RBC}, \mathrm{WBC}, \mathrm{NEU}$, and LYMP counts. $^{6}$

- Lot-to-lot variability from evacuated tubes: Lippi et al evaluated the filling of three different lots from three different sodium citrate evacuated tubes producers. Briefly, Greiner tubes were the most accurate (bias, -1.0 to $2.4 \%$ ), followed by Kima (bias, -7.8 to $-5.9 \%$ ), and Becton Dickinson (bias, -9.6 to 3.3\%) tubes. The highest betweenlot difference was noted for Becton Dickinson tubes (up to $12.9 \%$ ), followed by Greiner and Kima tubes (up to 3.4 and $1.8 \%$, respectively). ${ }^{37}$

Manufacturers of evacuated tubes worldwide commercialize three ethylenediaminetetraacetic acid (EDTA) preparations: $\mathrm{Na}_{2}$ EDTA, $\mathrm{K}_{2}$ EDTA, and $\mathrm{K}_{3}$ EDTA. The International Council for Standardization in Hematology recommended $\mathrm{K}_{2}$ EDTA (i.e., $1.5-2.2 \mathrm{mg} / \mathrm{mL}$ of blood) for hematological testing. ${ }^{38}$ However, laboratory professionals frequently select these evacuated tubes based on costs or local availability without considering that a change of tubes could be a source of laboratory variability. ${ }^{39,40}$ We decide to evaluate the impact of fasting time on $\mathrm{CBC}$ using both ( $\mathrm{K}_{2}$ EDTA and $\mathrm{K}_{3}$ EDTA) in two different analytical platforms (instruments) to provide scientific evidence mirroring the "real life" in different laboratories. Moreover, the direction of interference of lipemia varies relative to parameter and analytical platform. Furthermore, the degree of bias due to the nonfasting state depends on many different mechanisms (patient metabolism, body composition, rate of food absorption, type of food, etc.). ${ }^{28}$ Our results have shown significant differences between $K_{2}$ EDTA and $\mathrm{K}_{3}$ EDTA for Hct and MCV (-Table 2; - Fig. 1C, D). The differences observed ( $\mathbf{- T a b l e ~ 2 ; ~ - ~ F i g . ~} \mathbf{1}$ ) between different instruments comparing $K_{2}$ EDTA versus $K_{3}$ EDTA were previously described. ${ }^{41,42}$ Obviously, different combinations between evacuated tubes and instruments could be verified by each laboratory, as required during the accreditation process. $^{3}$

It is recognized that a fasting state prior to testing is not always possible or achievable, especially in emergency settings and inpatients receiving continuous parenteral nutritional support. Therefore, clinicians should be aware of the fasting state of the patient when interpreting these diagnostic results.

When looking at the above results, these parameters might be regarded as clinically irrelevant. However, such a conclusion would be wrong with respect to the current quality specifications for bias, derived from biological variation (-Fig. 1). Quality managers of medical laboratories consider the quality specifications derived from biological variation $^{43}$ both very important and useful in daily practice. $^{44}$ Moreover, we are waiting anxiously for the new database regarding biological variation that are under construction on behalf of the European Federation of Laboratory Medicine. $^{45}$

In conclusion, the significant variations observed in several hematological parameters, and aPTT due to breakfast feeding demonstrate that the fasting time needs to be carefully considered prior to performing routine hematological and coagulation testing to avoid interpretive mistakes of test results, and to guarantee patient safety. Due to the abovementioned reasons, the establishment of a mathematical correction through an algorithm directed to avoid fasting time is impossible and not recommended. Therefore, COLABIOCLI WG-PRE-LATAM encourages general practitioners, nurses, technicians, and laboratory professionals to operate all together to reduce laboratory variability and to standardize the fasting requirements in their laboratory (i.e., 12 hours) using the evidence reported above.

\section{Funding}

None declared.

Conflict of Interest

None declared. 


\section{Acknowledgments}

Our sincere thanks to Ana Pinilla for her dedication in collecting all blood samples. Our special thanks to Karina Olivares for the recruitment of all volunteers, and logistics coordination. Our grateful thanks to Claudia Gómez and Olaya Peñaloza for they data tabulation from internal quality control. Our gratitude to Riccardo Fiore (F.L. Medical, Torreglia, Italy) who partially supported the project ADR 2451/15.

\section{References}

1 Lima-Oliveira G, Volanski W, Lippi G, Picheth G, Guidi GC. Preanalytical phase management: a review of the procedures from patient preparation to laboratory analysis. Scand J Clin Lab Invest 2017;77(03):153-163

2 Lima-Oliveira G, Lippi G, Salvagno GL, Picheth G, Guidi GC. Laboratory diagnostics and quality of blood collection. J Med Biochem 2015;34(03):288-294

3 International Organization for Standardization. Medical Laboratories - Requirements for Quality and Competence ISO Document 15189. Geneva, Switzerland: International Organization for Standardization; 2012

4 Lippi G, Lima-Oliveira G, Salvagno GL, et al. Influence of a light meal on routine haematological tests. Blood Transfus 2010;8(02): 94-99

5 Simundic AM, Bölenius K, Cadamuro J, et al; Working Group for Preanalytical Phase (WG-PRE), of the European Federation of Clinical Chemistry and Laboratory Medicine (EFLM) and Latin American Working Group for Preanalytical Phase (WG-PRE-LATAM) of the Latin America Confederation of Clinical Biochemistry (COLABIOCLI). Joint EFLM-COLABIOCLI recommendation for venous blood sampling. Clin Chem Lab Med 2018;56(12):2015-2038

6 Lima-Oliveira G, Guidi GC, Salvagno GL, Danese E, Montagnana M, Lippi G. Patient posture for blood collection by venipuncture: recall for standardization after 28 years. Rev Bras Hematol Hemoter 2017;39(02):127-132

7 Lippi G, Salvagno GL, Lima-Oliveira G, Danese E, Favaloro EJ, Guidi GC. Influence of posture on routine hemostasis testing. Blood Coagul Fibrinolysis 2015;26(06):716-719

8 Lima-Oliveira G, Lippi G, Salvagno GL, et al. Transillumination: a new tool to eliminate the impact of venous stasis during the procedure for the collection of diagnostic blood specimens for routine haematological testing. Int J Lab Hematol 2011;33(05): 457-462

9 Lima-Oliveira G, Salvagno GL, Lippi G, et al. Elimination of the venous stasis error for routine coagulation testing by transillumination. Clin Chim Acta 2011;412(15-16):1482-1484

10 Lima-Oliveira G, Lippi G, Salvagno GL, et al. Venous stasis and whole blood platelet aggregometry: a question of data reliability and patient safety. Blood Coagul Fibrinolysis 2015;26(06):665668

11 Lima-Oliveira G, Guidi GC, Salvagno GL, Lippi G. The impact of fist clenching and its maintenance during venipuncture on routine hematology testing. J Clin Lab Anal 2017;31(05):31

12 Lippi G, Lima-Oliveira G, Guidi GC. Does fist pumping/clenching during venipuncture activate blood coagulation? Blood Coagul Fibrinolysis 2016;27(03):357-358

13 Lima-Oliveira G, Lippi G, Salvagno GL, Brocco G, Guidi GC. In vitro diagnostic company recalls and medical laboratory practices: an Italian case. Biochem Med (Zagreb) 2015;25(02):273-278

14 Simundic AM. Practical recommendations for statistical analysis and data presentation in Biochemia Medica journal. Biochem Med (Zagreb) 2012;22(01):15-23

15 Westgard J. Desirable Biological Variation Database Specifications. Available at: http://www.westgard.com/biodatabase1. htm2014. Accessed August 12, 2019
16 Matsuzawa T, Sakazume M. Effects of fasting on haematology and clinical chemistry values in the rat and dog. Comp Haematol Int 1994:4:152-156

17 Unger G, Benozzi SF, Campion A, Pennacchiotti GL. Preanalytical phase: effects of water ingestion during fasting on routine hematological parameters in a small cohort of young women. Clin Chim Acta 2018;483:126-129

18 Niv Y, Fraser GM. The alkaline tide phenomenon. J Clin Gastroenterol 2002;35(01):5-8

19 Rune SJ. The metabolic alkalosis following aspiration of gastric acid secretion. Scand J Clin Lab Invest 1965;17(04):305-310

20 Rune SJ. Comparison of the rates of gastric acid secretion in man after ingestion of food and after maximal stimulation with histamine. Gut 1966;7(04):344-350

21 Martin HE. Physiological leucocytosis: II. Post-prandial leucocytosis and Widal's haemoclasic test for hepatic efficiency. J Physiol 1932;77(01):63-67

22 Van Oostrom AJ, Sijmonsma TP, Rabelink TJ, Van Asbeck BS, Cabezas MC. Postprandial leukocyte increase in healthy subjects. Metabolism 2003;52(02):199-202

23 Mohamed-Ali V, Goodrick S, Rawesh A, et al. Subcutaneous adipose tissue releases interleukin-6, but not tumor necrosis factor-alpha, in vivo. J Clin Endocrinol Metab 1997;82(12): 4196-4200

24 Khan IM, Pokharel Y, Dadu RT, et al. Postprandial monocyte activation in individuals with metabolic syndrome. J Clin Endocrinol Metab 2016;101(11):4195-4204

25 Motton DD, Keim NL, Tenorio FA, Horn WF, Rutledge JC. Postprandial monocyte activation in response to meals with high and low glycemic loads in overweight women. Am J Clin Nutr 2007;85 (01):60-65

26 Klop B, van de Geijn GJ, NjoTL, et al. Leukocyte cell population data (volume conductivity scatter) in postprandial leukocyte activation. Int J Lab Hematol 2013;35(06):644-651

27 Cordova CMM, Galgowski C. Flexibilization of fasting for laboratory determination of the lipid profile in Brazil: science or convenience? Arq Bras Cardiol 2018;111(05):747-749

28 Guidi GC, Simundic AM, Salvagno GL, Aquino JL, Lima-Oliveira G. To avoid fasting time, more risk than benefits. Clin Chem Lab Med 2015;53(10):e261-e264

29 Hyson DA, Paglieroni TG, Wun T, Rutledge JC. Postprandial lipemia is associated with platelet and monocyte activation and increased monocyte cytokine expression in normolipemic men. Clin Appl Thromb Hemost 2002;8(02):147-155

30 Kälsch T, Elmas E, Nguyen XD, et al. Effects of alimentary lipemia and inflammation on platelet CD40-ligand. Thromb Res 2007;120 (05):703-708

31 Eikelboom JW, Hirsh J. Monitoring unfractionated heparin with the aPTT: time for a fresh look. Thromb Haemost 2006;96(05): 547-552

32 Nazari SSH, Mansournia MA. Case-crossover and case-time-control studies: concepts, design, and analysis. J Biostat Epidemiol 2015;1:59-69

33 Jensen AK, Gerds TA, Weeke P, Torp-Pedersen C, Andersen PK. On the validity of the case-time-control design for autocorrelated exposure histories. Epidemiology 2014;25(01):110-113

34 Lima-Oliveira G, Lippi G, Salvagno GL, Montagnana M, Picheth G, Guidi GC. The effective reduction of tourniquet application time after minor modification of the CLSI H03-A6 blood collection procedure. Biochem Med (Zagreb) 2013;23(03):308-315

35 Lima-Oliveira G, Lippi G, Salvagno GL, Montagnana M, Picheth G, Guidi GC. Impact of the phlebotomy training based on CLSI/NCCLS H03-a6 - procedures for the collection of diagnostic blood specimens by venipuncture. Biochem Med (Zagreb) 2012;22(03): 342-351

36 Lima-Oliveira G, Guidi GC, Salvagno GL, et al. Is phlebotomy part of the dark side in the clinical laboratory struggle for quality? Lab Med 2012;43:172-176 
37 Lippi G, Salvagno GL, Radišić Biljak V, et al. Filling accuracy and imprecision of commercial evacuated sodium citrate coagulation tubes. Scand J Clin Lab Invest 2019;79(04):276-279

38 Recommendations of the International Council for Standardization in Haematology for Ethylenediaminetetraacetic Acid Anticoagulation of Blood for Blood Cell Counting and Sizing. International Council for Standardization in Haematology: Expert Panel on Cytometry. Am J Clin Pathol 1993;100(04):371-372

39 Lima-Oliveira G, Lippi G, Salvagno GL, et al. Brand of dipotassium EDTA vacuum tube as a new source of pre-analytical variability in routine haematology testing. Br J Biomed Sci 2013;70(01):6-9

40 Lima-Oliveira G, Lippi G, Salvagno GL, et al. K(3)EDTA vacuum tubes validation for routine hematological testing. ISRN Hematol 2012;2012:875357

41 Lewis SM, England JM, Rowan RM. Current concerns in haematology. 3: blood count calibration. J Clin Pathol 1991;44(11):881-884
42 Goossens W, Van Duppen V, Verwilghen RL. K2- or K3-EDTA: the anticoagulant of choice in routine haematology? Clin Lab Haematol 1991;13(03):291-295

43 Ricós C, Alvarez V, Cava F, et al. Current databases on biological variation: pros, cons and progress. Scand J Clin Lab Invest 1999;59 (07):491-500

44 Ricós C, Cava F, García-Lario JV, et al. The reference change value: a proposal to interpret laboratory reports in serial testing based on biological variation. Scand J Clin Lab Invest 2004;64(03):175-184

45 Coskun A, Braga F, Carobene A, et al; European Federation of Clinical Chemistry and Laboratory Medicine (EFLM) Working Group on Biological Variation and Task Group for the Biological Variation Database. Systematic review and meta-analysis of within-subject and between-subject biological variation estimates of 20 haematological parameters. Clin Chem Lab Med 2020;58(01):25-32 